\title{
The scale of Gujarat's mortality crisis
}

Analysis of excess deaths from the civil registration system spotlights the systematic obfuscation in official statements

\section{Aashish Gupta \& Murad Banaji ${ }^{1}$}

By all accounts, the mortality impact of the COVID-19 pandemic has been exceptionally large. Crematoria, burial grounds, and, in some places, even riverbeds are full. Tragically, almost everyone has lost at least one person close to them. Given this reality, few people have much faith in official COVID-19 death counts. How many COVID-19 deaths are going uncounted? Could indirect effects of the pandemic, for example through economic distress and disruptions to health care, be contributing to a surge in mortality?

\section{Registration data insights}

These key questions, being asked for over a year, are even more pertinent now. In Gujarat, reports of overburdened crematoria and lengthy lists of obituaries indicate a severe crisis. Mortality figures from the civil registration system in Gujarat, reported recently by the Gujarati newspaper, Divya Bhaskar (https://bit.ly/3oNDnjw), provide some insight into the scale of the mortality crisis.

To provide some context, official COVID death-counts largely reflect deaths in hospitals, of patients with confirmed COVID-19, where COVID-19 is listed as a cause of death. Deaths where doctors do not write COVID-19 as a cause of death on the hospital death certificate are missed. Additionally, government "death audit committees" may choose to omit deaths of patients with confirmed COVID-19 from official tolls, citing co-morbidities as the main cause of death. Finally, deaths at home, whether of those who were tested or not, and whether test results were positive or not, are largely missed by the system.

In contrast, India's civil registration system is supposed to register all deaths - COVID or not, at home or in a hospital. Civil registration has been improving, and across India about $86 \%$ of all deaths were registered in 2018. In Gujarat, civil registration is considered to be complete by the Registrar General and Census Commissioner of India (https://bit.ly/3wt1Ahx).

Divya Bhaskar reported that during a 71-day period between March 1, 2021 and May 10, 2021, there were 1.23 lakh deaths registered in Gujarat. In the corresponding period in 2020, there were around 58,000 registered deaths. This suggests a large increase in mortality in 2021.

\footnotetext{
${ }^{1}$ Aashish Gupta (aashishg@sas.upenn.edu) is a PhD candidate in Demography and Sociology at the University of Pennsylvania, U.S. Murad Banaji (m.banaji@mdx.ac.uk) is a Senior Lecturer in Mathematics at Middlesex University London. A version of this short note appeared in The Hindu (page 6, 25 ${ }^{\text {th }}$ May 2021).
} 


\section{Year-wise comparison}

However, 2020 was also a pandemic year, and the early days of the pandemic saw a very harsh lockdown. This likely prevented or delayed many deaths from being registered. Considering this, we compared registered deaths in Gujarat in 2021 to estimates of baseline mortality from previous years.

The Sample Registration System, a continuous demographic survey, provides estimates of crude death rates in Gujarat until 2018. For the period 2015-2018, mean crude death rates were 6.075 deaths per 1,000. Taking Gujarat's projected population in 2021, about 6.98 crore, we would expect about 82,500 deaths in a 71-day period. Using registered deaths between 2015-2018 for this calculation reveals a similar estimate.

The 1.23 lakh registered deaths in Gujarat during March 1-May 10, 2021 were thus 50\% higher than expected from baseline estimates. Moreover, the current COVID-19 wave may have caused delays in registration - the scale of the mortality surge could be even greater.

Thus, in a little over two months, there were at least 40,000 "excess deaths" over and above what we expect from previous years' data. This is around 10 times Gujarat's official COVID-19 death count during this period of around 4,200 deaths.

The increase in excess mortality mirrors the timing of the rise in recorded COVID-19 deaths, which rose sharply through most of April. This can be seen in the graph of annualised crude death rates. But we cannot assume that all Gujarat's excess deaths are COVID-19 deaths. Some may reflect overwhelmed health-care facilities or worsening economic conditions. In fact, there is some evidence for possible indirect effects. The Divya Bhaskar report documents that $20 \%$ of the deaths that were registered were among people less than 25 years of age. This is an increase in both absolute and relative terms compared to 2018 , when about $15 \%$ of deaths were below the age of 25. Because young people are less vulnerable to severe COVID-19, and because child mortality is especially sensitive to disruptions in health care, this suggests an increase in indirect mortality.

Even factoring in a possible increase in indirect mortality, the data strongly suggest that the great majority of COVID-19 deaths in the State have gone uncounted. This is consistent with large mismatches observed between numbers of COVID-protocol cremations and recorded COVID-19 deaths.

The work of journalists in Gujarat demonstrates the value of looking at mortality records from civil registration to examine the extent of the crisis. In addition to Gujarat, civil registration is complete in 15 States and Union Territories. Many people work hard to collect this data, and many more wait in government offices to report the deaths of their family members. The least governments can do is to make this data available so that we can better understand the scale of India's COVID-19 crisis. 
Figure 1: Large increase observed crude death rates in Gujarat when compared to estimates of expected baseline mortality

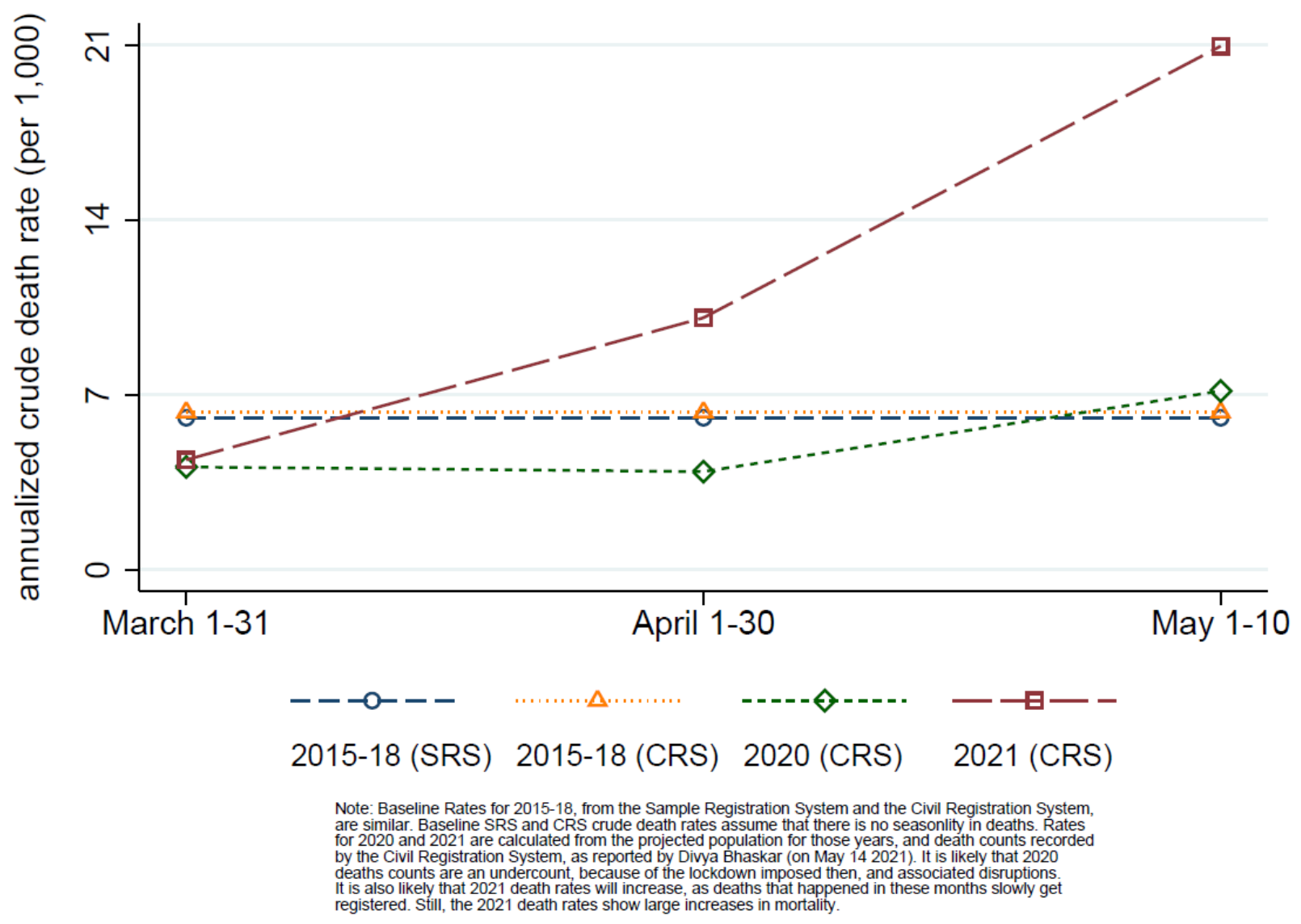

\section{Unreliability of official counts}

In summary, data from the civil registration system in Gujarat shows large increases in mortality, consistent with multiple accounts of the unreliability of official COVID-19 death reporting in the State. These official death counts are used to make claims about low infection fatality rates and low overall mortality. Analysis of excess deaths from the civil registration system highlights the systematic obfuscation in such official statements.

Ultimately, Gujarat's civil registration system death counts reinforce what we already know that an unprecedented tragedy is unfolding. And that the government's response has been severely lacking. 